\title{
Impact of the COVID-19 Pandemic on Clinical Trial Conduct: Lessons for the Future
}

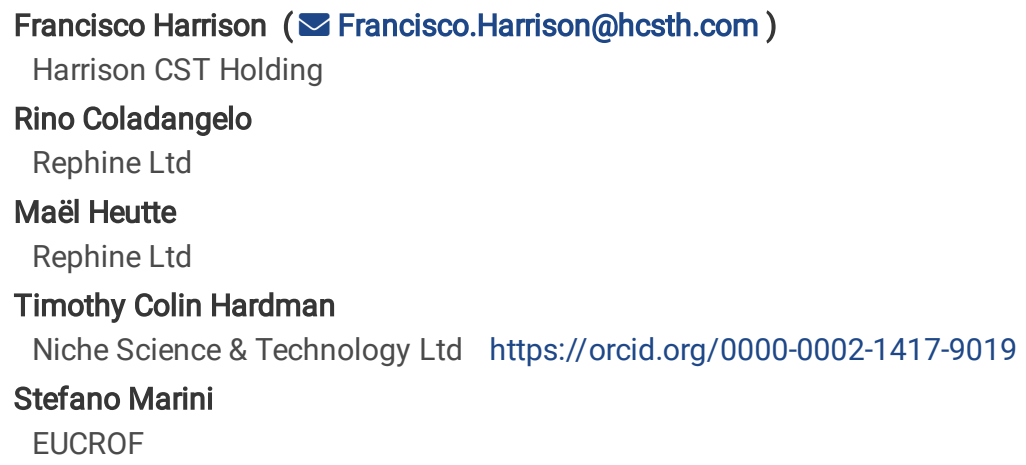

\section{Research Article}

Keywords: COVID-19, pandemic, clinical trial, contract research organisation, CRO

Posted Date: February 14th, 2022

DOI: https://doi.org/10.21203/rs.3.rs-1118410/v1

License: (c) (i) This work is licensed under a Creative Commons Attribution 4.0 International License. Read Full License 


\section{Abstract}

Background: The COVID-19 outbreak was declared to be a pandemic by the WHO in March 2020. Ongoing clinical trials were suspended, some never to re-start. A survey was conducted to get a better understanding of both the successes and failures in dealing with the pandemic.

Methods: European contract research organisations (CROs) were asked to determine the extent to which the pandemic impacted on their operations in terms of the trial site conduct and investigator performance as well as its impact on subjects and patients involved in ongoing trials and the quality of data generated.

Results: Forty-nine companies provided responses to the questionnaire. Data indicated that the challenges faced during the pandemic had a marked impact on clinical trial conduct and delivery. We saw an almost immediate decline in clinical trial activity associated with reduced subject/patient recruitment and a reluctance of study participants to undergo follow-up due to their fears of exposure to infection. There was insufficient data to determine the full extent of the pandemic on clinical trial performance and associated limitations in access to bed spaces and the resources necessary to perform procedures and subject follow-up. The introduction of remote monitoring by CROs provided the opportunity for essential activities associated with clinical trials to resume/continue. However, implementing these solutions required research organisations to implement changes to trial documentation and procedures. This placed marked burdens on budgets and clinical resources.

Conclusions: Those involved in clinical trials advanced a flexible approach to addressing the challenges posed by COVID-19, with all parties working closely together to find solutions.

\section{Introduction}

Early in 2020, COVID-19 was spreading rapidly across the globe. The World Health Organisation declared the COVID-19 outbreak to be a pandemic on 11 March 2020 [1], having a marked effect on every aspect of our lives. Clinical trials were also impacted, with the consequence of the pandemic on clinical research believed to peak between March and May, 2020. While stakeholders assessed the impact of the pandemic, ongoing clinical trials were suspended, some never to re-start [1-6].

Despite players within the clinical trial space having established procedures within their quality systems to deal with service disruption in the form of 'disaster plans', it was apparent that many contract research organisations (CROs) were not prepared adequately for the restrictions that affected patients/subjects, sites and investigators and monitoring activities. Information sourced from databases such as Global Data suggested that by March 2020, more than sponsor 500 companies and 1,000 CROs were publicly admitting to disruptions to their planned and ongoing clinical trials [7]. The number of studies affected reportedly increased steadily during the last quarter of 2020 [8]. The clinical trial community needed to innovate to address service interruptions. Industry players introduced new, pragmatic changes to operational procedures to allow businesses and clinical studies to continue running during the COVID-19 outbreak. The current survey was conducted during the second half of 2020 to get a better understanding of both the successes and failures in dealing with the pandemic.

\section{Methods}

The European CRO Federation (EUCROF) consists of Members from 12 EU countries (Belgium (BeCRO), Czech Republic (ACRO-CZ), France (AFCROs), Germany (BVMA), Greece (HACRO), Italy (AICRO), Romania (ACCSCR), Slovakia (SACROP), Spain (AECIC), The Netherlands (ACRON), Turkey (SAKDER) and the United Kingdom (CCRA). The Federation has also 14 associate members in Albania, Austria, Bulgaria, Croatia, Denmark, Georgia, Serbia, Spain, Sweden, Switzerland (2), Ukraine and the United Kingdom (2), as well as three partner members in Algeria, Egypt and Israel. At the start of the pandemic EUCROF represented 387 member organisations across 25 countries, employing approximately over 25.000 clinical and administrative staff running hundreds of studies. All members were invited by email to take part in the survey, performed between 1 to the 31 October, 2020.

The survey included 20 multiple-choice, fixed-response questions and two free-text entry fields. It was designed to be completed in under 10 minutes. Participants were asked to evaluate the impact of the pandemic on key aspects relating to their performance in their clinical trials using a range of 1 (no-impact) to 10 (extremely serious) (see Appendix 1).

The questionnaire was distributed to member organisations by email and it is assumed any answers provided reflected the local impact of the pandemic at that particular moment.

\section{Data analysis}

Participant responses were collected automatically and exported into a Microsoft Excel spreadsheet. After harmonisation, quantitative data analysis was performed using IBM Statistics SPSS 25.5. The responses for each of the 'questions' were counted and calculated as a 
percentage. Where entries were not provided, the data fields were left blank. Free-text responses were scored according to the number of respondents mentioning specific words or points.

\section{Results \\ Respondents}

Forty-nine of the 387 member organisations from 15 countries provided completed responses to the survey. The greatest proportion of responders were from Germany (17\%) and France (15\%) (Fig. 1a).

\section{Immediate impact on clinical trial delivery}

No responders avoided trial disruption resulting from the pandemic measures, with $63 \%$ of the responders recording a marked or 'serious' on their ongoing trials and almost a third (30\%) noting that the impact was 'extremely serious' (Fig. 1c). Our data suggested that Phase III trials were more markedly affected than earlier phase trials.

Responses provided in the free text fields suggest that the impact was worse than the ordinate responses imply; $92 \%$ of responders reported that they had faced adverse challenges to their delivery models. However, several positive comments (four in total) infer that responder companies were able to introduce adaptations to the way they delivered their services, and continue conducting trials. Challenges can be divided into three main categories. The most frequently reported issue involved influence of the pandemic on hospital recruitment (42\% of responders), where investigators were no longer able to conduct trials and/or patients unwilling to make hospital visits. In $35 \%$ of cases, operational issues were the cause of disruption including delays in dispensing investigational medicines, initiation of site visits and issues around laboratory services (processing samples). The remaining issues (20\%) involved those related to monitoring, such as limited monitor access to centres, files or investigators.

\section{Trial participation}

A broad sweep of national and local pandemic interventions, such as restrictions to travel and lockdowns, that limited the access that study participants and trial staff had to trial sites. This was probably a major reason why the pandemic markedly reduced the rate of recruitment and the availability of study participants to attend study starts, continue or even conclude their involvement in clinical trials. According to comments provided in the survey, participants skipped visits to the clinic fearing contamination which, in many cases, resulted in physicians being unable to recruitment or resume trials, or even perform closeout follow-up assessments. This drove sponsors, CROs, investigators and patient groups to explore alternative ways of resuming studies.

Many trials adopted a decentralised approach, following the US Food and Drug Administration (FDA) and the European Medicines Agency (EMA) COVID-19 specific guidelines provided as means of mitigating the risks of clinical trial participation. Using local rather than central services reduced the need for travel and minimised face-to-face contact [9-14]. Home/remote visits were also used. Other measures included implementation of strict patient protection and sanitation measures at site and novel channels for medication delivery. Although $53 \%$ of responders mentioned that the pandemic affected the patient's participation, $44 \%$ of responders (written comments) also noted the number of solutions being generated, to the completion of post-study visits and collection of data.

\section{Technology-driven trial management}

One aspect emerging from the survey was the role played by electronic signatures and electronic documentation particularly with regards to informed consenting of patients. Key issues surrounded the implementation of informed consent via electronic resources, which was introduced without any recognised standards that the industry should adhere to [15]. This could include gaining consent verbally on the telephone, tablet computers at study centres or other means of registering consent without physically signing documents. These techniques were generally wellreceived and it seems likely that electronic consenting will continue once the pandemic is over.

The use of remote meetings and video conferences was a key part of a technology transformation revolution. How CROs reacted to the challenges such plausibility checks, source data verification (SDV), query management or adverse event reporting is summarised in Fig. 2. Plausibility checks are remote in $30 \%$ of the CROs, finding alternative solutions such as video conferencing, scanning and telephone conferences to perform this activity.

\section{Paper records and missing data}

Remote monitoring and access to documentation belonging to Trial Master Files (TMFs), while maintaining advanced quality management formed a critical component of continued service delivery. The shift to remote and hybrid working underlined the dependence that many trials have on paper records. Respondents highlighted some of the limitations with remote data collection and it was clear that not all outputs were suitable for remote collection. Twenty-four per cent of responders reported the use of paper case report forms (CRFs) and 76\% reported using 
electronic (e)CRFs. The trend of using eCRFs which has become prevalent over the last decade, certainly aided the CRO industry in overcoming the present demands.

Source data verification processes were modified in more than $53 \%$ of the CROs (Fig. 2a). However, methods used to record data on adverse event episodes or the data queries remain unchanged. Written responses were mostly positive on the issue of how missing data have been addressed. Most (over 60\%) respondents did not plan to replace any participants for studies with an incomplete dataset and intend to analyse study data as they are. More patients will not be included in the studies than planned and studies will not be stopped or restarted unless the sponsor has instructed otherwise. The finding regarding the questions relating to how survey responders would address the missing data are summarised in Fig. 2b.

\section{Study monitoring}

Most survey participants indicated in their response that the quality of work had not suffered as a consequence of the pandemic. This was attributed to the introduction of new activities and tools, such as remote monitoring and the apparent increase in the frequency of communication with sites. The quality of monitoring via on site visits remained high (average score: 8.37; Fig. 3a), higher than that for 'remote' monitoring visits (average score: 6.04; Fig. 3b), as all objectives of the monitoring check lists could not be completed remotely.

On site monitoring was a key aspect of study conduct that was markedly affected by the pandemic. The introduction of stringent health checks at site restricted monitor access to documentation and the investigator teams. The overall impact was to make monitoring visits longer and more complex. The practical upshot was that the cost of conducting on site monitoring increased (increased time of the monitor on site and for the CRO managing the visits). In contrast, where remote monitoring was already being employed the impact of the pandemic appears to have been lower (Fig. 3c and Fig. 3d). However, a change in procedures to permit the adoption of remote monitoring by studies already running incurred additional costs with the introduction of alternate systems and technologies.

\section{Operational, regulatory and scope changes}

During the pandemic, $61 \%$ of respondents reported cases were protocols required amending, often a consequence of necessary changes to monitoring visit schedules, the introduction of remote SDV and/or risk management plans or study extension. A summary of methodological, operative, regulatory and changes is provided in Fig. 2c. In many cases, where decisions were made to continue rather than close studies prematurely, the operational and regulatory changes placed additional resource burdens on sites and CROs.

In the majority of cases budgets were maintained and no discounts requested (fewer than $20 \%$ ). In over $90 \%$ of cases there was close agreement between CROs and Sponsors on the introduction of modifications and how these would influence contractual situations and how they should be funded. Changes in contracts occurred in over $50 \%$ of studies and Sponsors fulfilled their obligations in almost all cases (over $76 \%$ ), only a quarter were reluctant or rejected requests to pay for the services provided. In the questionnaire, CROs were asked about the collaboration with sponsors, and how this evolved during the pandemic. Most agreed that a good relationship and a pragmatic approach was maintained throughout.

\section{Supply management}

Survey participants indicated that only $4 \%$ of studies could not deliver the essential requirement of drug delivery to sites/patients, whereas $46 \%$ adapted new strategies for the supply distribution chain to patient (Fig. 4).

\section{Discussion}

The present survey confirms the findings of others, that few clinical trials were able to avoid disruption during the pandemic, and yet although many faced temporary pauses or suspensions, all but a few were cancelled [16-18]. Estimates suggested that during the pandemic National Institute of Health $(\mathrm{NIH})$ funded research suffered losses of more than $\$ 10$ billion due to COVID-19, with over $85 \%$ of research projects being affected by either directly or indirectly [19]. In this report we have described some of the adaptations CROs explored in their pragmatic approach to the challenges posed by the pandemic. Not only were many of these adaptations successful but they have significant implications for the future conduct of trials.

It wasn't surprising that CROs indicated that Phase II and Phase III studies were the most heavily affected by the pandemic. Limitations on travel during the pandemic saw the decentralised approach come to the fore [20,21]. Some trials have used primary care sites, such as general practice clinics and pharmacies, to deliver interventions and/or conduct sample collection rather than hospitals or CRO sites. Home visits have been used to reduce the number of people study subjects need to come into contact with - which is likely to be a key retention factor when study conditions associated with weakened immune systems. Overall, the decentralised approach, which can also encompass online assessment, delivery of interventions and self-assessment has been considered as a means of widening recruitment options and boosting retention, two major concerns for all clinical trials [15, 20,21]. It seems reasonable to conclude that efforts to adopt more decentralised

Page $4 / 11$ 
strategies will continue post-pandemic; however, consideration needs to be given to concerns over data quality oversight, increased complexity to management and costs.

Methods of gaining consent remotely or electronically were viewed favourable by our respondents. Remote consenting certainly reduces the reliance on paper forms, which the pandemic has highlighted can be problematic. The validity of the electronic signature has been repeated and reconfirmed by comments and publications from the US FDA and the EMA $[13,22]$. There are concerns about the quality, regulatory compliance and especially the recognition of electronic signatures as the new standard. However, it is recognised that there are inefficacies inherent in many of the methods currently employed to record consent. This has created an impetus to identify new ways to address the situation and has shown clearly that the clinical research world is extremely innovative and ready to change and incorporate new approaches quickly. However, it has also been suggested that this increased flexibility might, once again, aid participant recruitment.

Both the FDA and EMA were aligned in that the primary concern remains patient safety and that all parties must maintain their efforts to avoid any unnecessary infection in populations that may already be at high risk. Both opened the door to the pragmatic use of electronic media and electronic signatures during the pandemic $[22,23]$ and even recommended remote monitoring visits (if applicable) via electronic channels. It was clear that this will change the way authorities judge trial format and will enable them in the future to oversee sites, investigators and eventually patients while trials are still in progress. Total transparency, digital procedures and remote control while maintaining the highest level of quality and accomplishment of the regulatory requirements are the milestones to achieve and maintain. There is still some way to go but the future is clear to see.

The necessary introduction of remote practices also created increased opportunity for team communication and collaboration. To minimise risk it was considered essential to conduct regular meetings between the whole trial team and investigators around protocol amendments and exploring mechanisms to facilitative the introduction of remote practices. Various conclusions were made from the answers provided by the survey participants which were that good communication allowed most respondents to address the issues of trial management quickly as issues arose. The use of remote meetings and video conferences has been a key part of a technology transformation revolution. Due to the pandemic, this transformation is expected to remain and will influence existing industry processes going forward [21]. However, concerns were voiced over the ability to maintain engagement across teams by virtual communications alone, particularly if communication is driven by emails. It is clear that there remains a role for face-to-face interactions, it could be argued that participants may feel more comfortable communicating in person or that certain social signals or nuances may be missed in video interactions. The adoption of new tools could influence the future internal need for qualified staff and specific training for these tools. This will improve and accelerate the speed of research assuming all parties agree and are involved, which in the long run will be highly cost effective.

For decades, the landscape and model for clinical trials necessitated on-site, in-person monitoring and auditing. Information on study participants and events were recorded on paper case report forms. Medical records were documented on paper. The only way that representatives of study sponsors, CROs and regulatory authorities could review such data was by traveling to study sites and manually combing through these records. Recent years have seen a remarkable technological shift and many clinical trial case report forms are now electronic. The COVID-19 pandemic appears to have provided the impetus to revisit the feasibility and utility of remote monitoring. Travel restrictions, quarantines, and safety concerns have prevented on-site monitoring activities at most sites. Along with other adjustments such as remote consent, telehealth visits, off-site diagnostic studies, and shipping of oral study therapy directly to patients' homes, the FDA, the National Institutes of Health Central Institutional Review Board, and the National Cancer Institute have also encouraged remote monitoring [6, 13]. COVID19-related changes have shown us that remote or virtual methods can allow completion of some study visits in a manner that is quicker, cheaper, and less burdensome, without sacrificing quality.

A major proportion of our respondents found it necessary to make adaptations to their trial procedures as has been reported elsewhere [2- 6]. Amending trial protocols is time consuming and places a significant burden on study budgets [24, 25]. It is clear that the timelines for approval of amendments is currently unpragmatic and can result in subjects needing to stop receiving treatment and loss of primary study data. It may also result in sponsor procrastination because they anticipate that approval by regulatory and ethical bodies will be protracted. Considering the move to more adaptive approaches to trial protocols it seems reasonable to predict that future trial protocols may involve more pragmatic language and increased flexibility to reduce the number of amendments. However, it is essential that any modifications in trial delivery are introduced robustly across the board to avoid protocol deviations.

A major area of change was that of supply management; having to make the 'extra mile' in the real meaning of the word to bring the drug supplies to some patients enrolled in trials [26]. The changes and loss of the planned study delivery pathway forced many managers to consider how to make drugs and test equipment and other necessary tools and materials available to patients while maintaining Good Clinical Practice compliance and abiding by the ethical and confidentiality guidelines. This situation also underlined the importance of external service providers dedicated to help accomplish this goal. A very small proportion our participants (4\%) indicated that only $4 \%$ of studies could not deliver the essential requirement of drug delivery to sites/patients, whereas nearly half adapted new strategies for the supply distribution chain to patient. 
Sponsors appeared to have adopted a pragmatic approach to the burden placed on budgets and resources imposed by the pandemic. Even when studies were paused or cancelled there were few calls for budget adjustments. In general, sponsors and service providers maintained close communications which appeared to be positive in most cases noting just a few exceptions (only four negative remarks). Sponsors were amenable to increased requirements that CROs faced in attempting to keep studies running. Good communication appeared the key factor in the successful introduction and adoption of alternate methods and novel tools to ensure study delivery. Where Sponsors attempted to evaluate novel methods of trial delivery a common theme to arise was whether an approach shifted a burden or challenge from one aspect of a trial to another. CROs were sensitive to the potential that seemingly beneficial approaches may displace costs and create potentially unseen burden elsewhere.

Consideration of our survey data leads to the conclusion that those involved in clinical trials during the pandemic advanced a flexible approach to addressing the challenges posed by COVID-19, with all parties working closely together to find solutions. A range of innovative and pragmatic practices for managing and running clinical trials were introduced. It has been proposed that the pandemic has been the axis point for the industry and that many of our adaptations will become part of future working practices. The pandemic has also highlighted areas where 'structural' barriers to the way we conduct studies make it difficult to adopt innovation. It will be important in the upcoming months and years to evaluate/audit and validate the methods we have employed before new regulations and guidance can be provided.

\section{Declarations}

\section{ETHICS}

No ethical approval was sought for this study.

\section{ACKNOWLEDGEMENTS}

The authors would like to acknowledge the contribution that each of the member organisations made in responding to the survey. As the corresponding author, Dr Harrison has agreed to be accountable for all aspects of the work in ensuring that questions related to the accuracy or integrity of any part of the work are appropriately investigated and resolved.

\section{DISCLOSURE \& CONSENT}

This survey was performed with the support of the European Contract Research Organisation Federation (EUCROF) and polled their 385 members from across 22 European countries, including Israel and Algeria. The authors state they have no competing interests. All authors give consent for the current data and manuscript to be published

\section{FUNDING}

The authors received no financial support for the research, authorship, and/or publication of this article.

\section{AUTHORS' CONTRIBUTIONS}

All authors were involved in devising and completing the current work in addition to the analysis and writing of the final manuscript.

\section{References}

1. WHO Director-General's opening remarks at the media briefing on COVID-19 [Internet]. WHO.2020. https://www.who.int/directorgeneral/speeches/detail/who-director-general-s-opening-remarks-at-the-media-briefing-on-covid-19--11-march-2020 [Accessed 05 June 2021]

2. Asaad M, Habibullah NK, Butler CE. The Impact of COVID-19 on Clinical Trials. Ann Surg 2020;272:e222-e223.

3. Hasford J. Impact of the COVID-19 pandemic on clinical trials with drugs, Expert Opinion on Drug Safety, 2020:19:11; 1373-1375, DOI: 10.1080/14740338.2020.1828861

4. Early Impact of COVID-19 on the Conduct of Oncology Clinical Trials and Long-Term Opportunities for Transformation: Findings From an American Society of Clinical Oncology Survey.

5. Waterhouse, DM, Harvey RD, Hurley P, et al. Challenges in Assessing the Impact of the COVID-19 Pandemic on the Integrity and Interpretability of Clinical Trials. Stat Biopharm Res, 2020:12:4, 419-426, DOI: 10.1080/19466315.2020.1788984

6. Kunz CU, Jörgens S, Bretz F, et al. Clinical Trials Impacted by the COVID-19 Pandemic: Adaptive Designs to the Rescue?, Stat Biopharm Res, 2020: 12:4, 461-477, DOI: 10.1080/19466315.2020.1799857

7. Global Data Coronavirus Disease COVID19 PharmaExecutive Briefing [Internet] GlobalData. 2021. www.globaldata.com [Accessed 01 June 2021]

Page 6/11 
8. QPS. How COVID-19 Has Impacted Clinical Trials [Internet] 2021. https://www.qps.com/2021/02/17/how-covid-19-has-impacted-clinicaltrials/ [Accessed 05 June 21]

9. US Food and Drug Administration: FDA guidance on conduct of clinical trials of medical products during COVID-19 pandemic: Guidance for industry, investigators, and institutional review boards. https://www.fda.gov/media/136238/download [Accessed 03 June 2021]

10. Sidley.com: Considerations for sponsors of ongoing clinical trials in light of COVID-19 (part 1).

https://www.sidley.com/en/insights/newsupdates/2020/03/covid19-considerations-for-sponsors-of-ongoing-clinical-trials-in-light-ofcovid19-part1 [Accessed 03 June 2021]

11. Mooney M, McCaskill-Stevens W: Interim Guidance for Patients on Clinical Trials Supported by the NCI Cancer Therapy Evaluation Program and the NCl Community Oncology Research Program (NCORP), 2020.

https://ctep.cancer.gov/content/docs/Memorandum_on_Interim_Guidance_for_Clinical_Trial_Activities_Affected_by_the_Novel_Coronavirus3-13-2020.pdf [Accessed 03 June 2021]

12. Clinical Trials Transformation Initiative: Webinar recording available: Listen to best practices on conducting clinical trials during COVID-19. https://www.ctticlinicaltrials. org/news/webinar-recording-available-listen-best-practices-conducting-clinical-trials-during-covid-19 [Accessed 03 June 2021]

13. FDA Guidance on Conduct of Clinical Trials of Medical Products During the COVID-19 Public Health Emergency [Internet]. US Food \& Drug Administration 2020 https://www.fda.gov/regulatory-information/search-fda-guidance-documents/fda-guidance-conduct-clinical-trialsmedical-products-during-covid-19-public-health-emergency [Accessed 04 June 2021]

14. Guidance for medicine developers and other stakeholders on COVID-19. [Internet] European Medicines Agency 2020 https://www.ema.europa.eu/en/human-regulatory/overview/public-health-threats/coronavirus-disease-covid-19/guidance-medicinedevelopers-other-stakeholders-covid-19 [Accessed 03 June 2021]

15. Cindy Chen, Lee P, Pain KJ, Delgado D, Cole CL., Campion Jr TRC. Replacing Paper Informed Consent with Electronic Informed Consent for Research in Academic Medical Centers: A Scoping Review. [Internet] AMIA Jt Summits Trans/ Sci Proc [cited 2021 May 23 ] 80-88. https://pubmed.ncbi.nlm.nih.gov/32477626/ [Accessed 02 June 2021]

16. Park JJH, Mogg R, Smith GE, et al. How COVID-19 has fundamentally changed clinical research in global health. Lancet Glob Health 2021; 9: e711-20

17. Ledford H. Coronavirus shuts down trials of drugs for multiple other diseases. Nature. 2020;580:15-16.

18. Upadhaya S, Yu JX, Oliva C, et al. Impact of COVID-19 on oncology clinical trials. Nat Rev. 2020;19:377-378.

19. NIH Director Dr. Francis Collins Testifies Loss of \$10 Billion in Research Loss Due to COVID-19. [Videoclip. C-Span.] 2020. https://www.cspan.org/video/?c4890754/user-clip-nih-director-dr-francis-collins-testifies-loss-10-billion-research-loss-due-covid-19 [Accessed 04 June 2021]

20. Miseta, E., Kasher, J., Lipset, C. COVID-19 Hastens Embrace Of Virtual 2020. Clinical Leader., https://www.clinicalleader.com/doc/covidhastens-embrace-of-virtual-trials-0001 [Accessed 07 June 2021]

21. Why COVID-19 is Accelerating Technology Transformation in Clinical Research [Internet] Florence. 2020 November. https://florencehc.com/blog-post/why-covid-19-is-accelerating-technology-transformation-in-clinical-research/ [Accessed 07 June 2021]

22. E-signatures clarified in FDA's COVID-19 clinical trial guidance [Internet] Regulatory Affairs Professional Society. 2020. https://www.raps.org/news-and-articles/news-articles/2020/6/e-signatures-clarified-in-fdas-covid-19-clinical-t [Accessed 06 June 2021]

23. Guidance on the management of clinical trials during the COVID-19 Pandemic [Internet] European Medicines Agency 2021. https://ec.europa.eu/health/sites/default/files/files/eudralex/vol-10/guidanceclinicaltrials_covid19_en.pdf. [Accessed 08 June 2021]

24. Getz A. Protocol Amendments: a Costly Solution. Applied Clinical Trials 2011, 20.

25. Getz A, Stergiopoulos S, Short M, et al. The Impact of Protocol Amendments on Clinical Trial Performance and Cost. Therap Innovation Reg Sci 2016:50(4): DOI: 10.1177/2168479016632271

26. Decentralised trials call for supply chain: Marken [Internet]. Spinner J. Outsourcing Pharma.com. 2021. https://www.outsourcingpharma.com/Article/2021/04/12/Decentralized-trials-call-for-supply-chain-flexibility [Accessed 08 June 2021]

27. National Institutes of Health: Guidance for NIH-Funded Clinical Trials and Human Subjects Studies Affected by COVID-19. 2020. www.grants.nih.gov/grants/guide/notice-files/NOT-OD-20-087.html

\section{Figures}


(a)

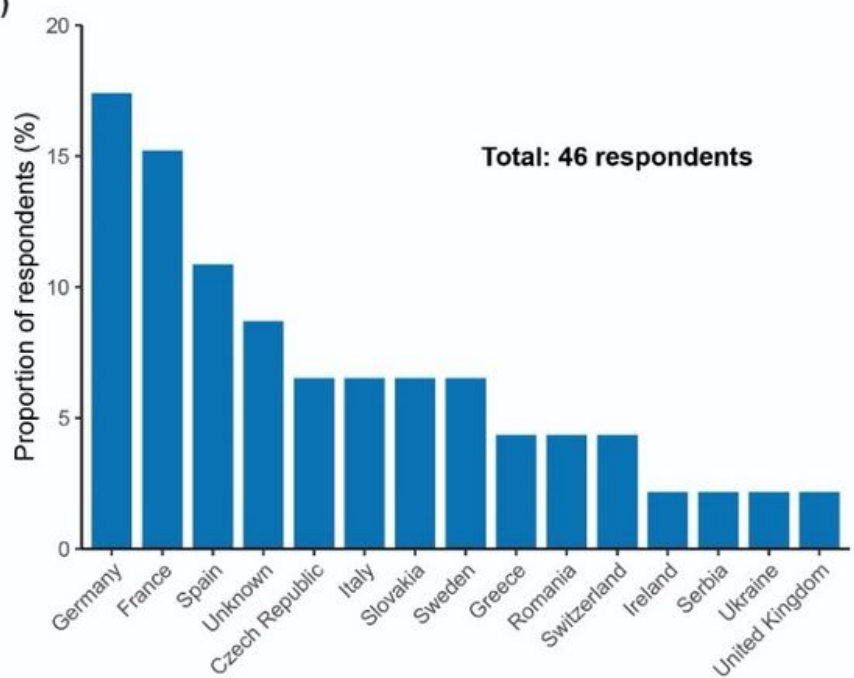

(c)

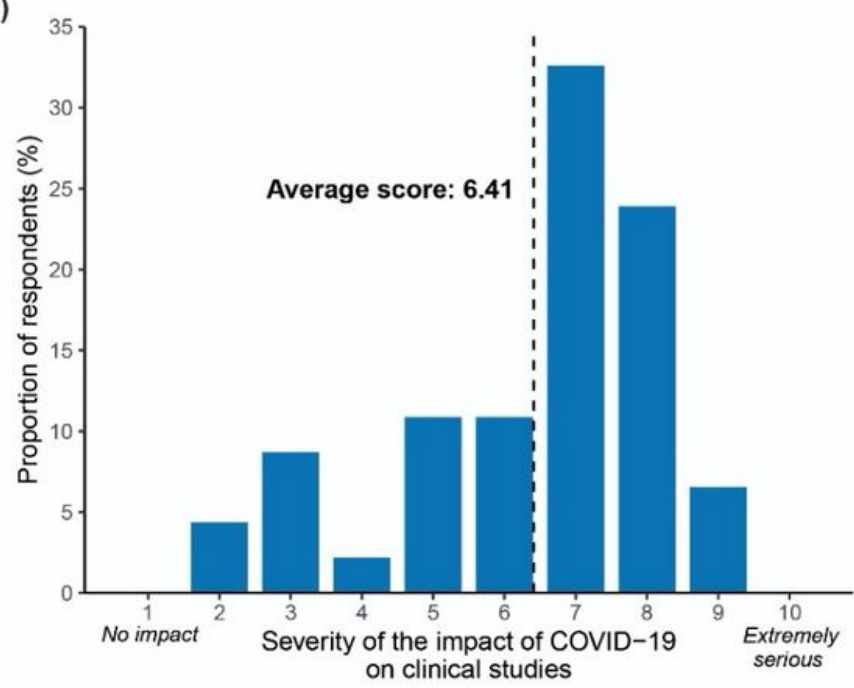

(b)

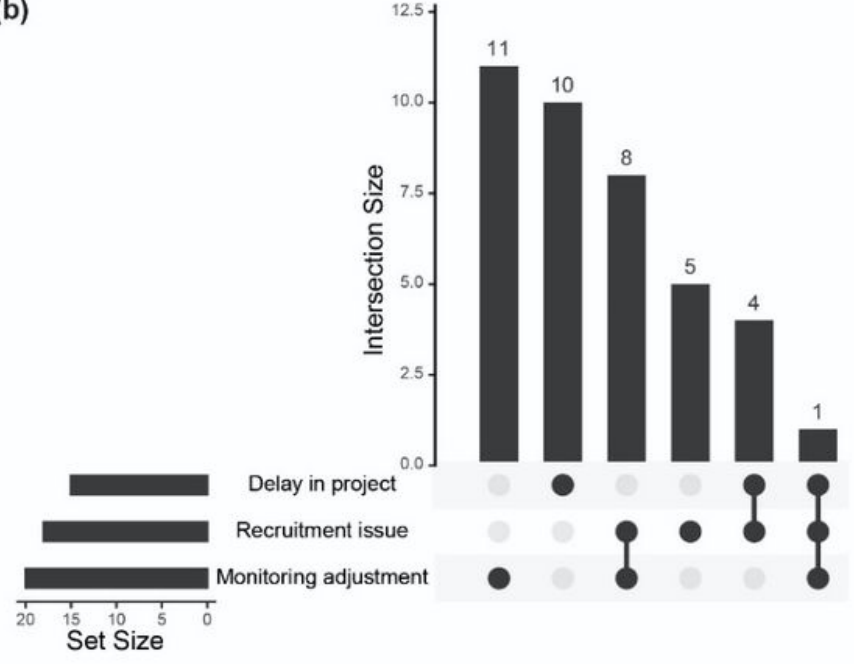

(d)



Figure 1

Overall impact of COVID-19 on clinical trials assessed from the survey. (a) Distribution of the respondents by country of origin. (b) Main effects of COVID-19 on the clinical studies. (c and d) Severity of the impact of COVID-19 ranked on a 1-10 scale, 1 being "no impact" and 10 "extremely serious", (c) on clinical studies and (d) on patients. 
(a)

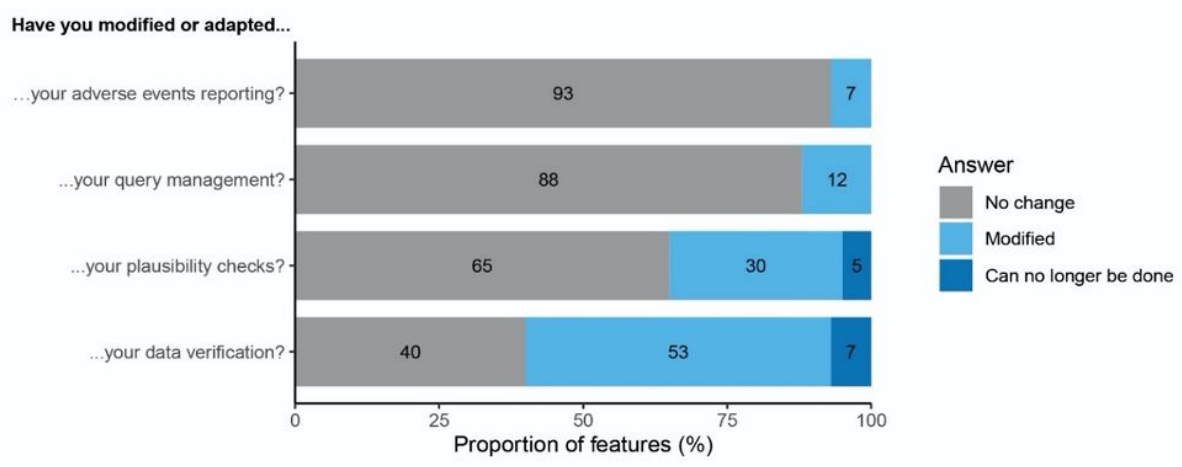

(b)

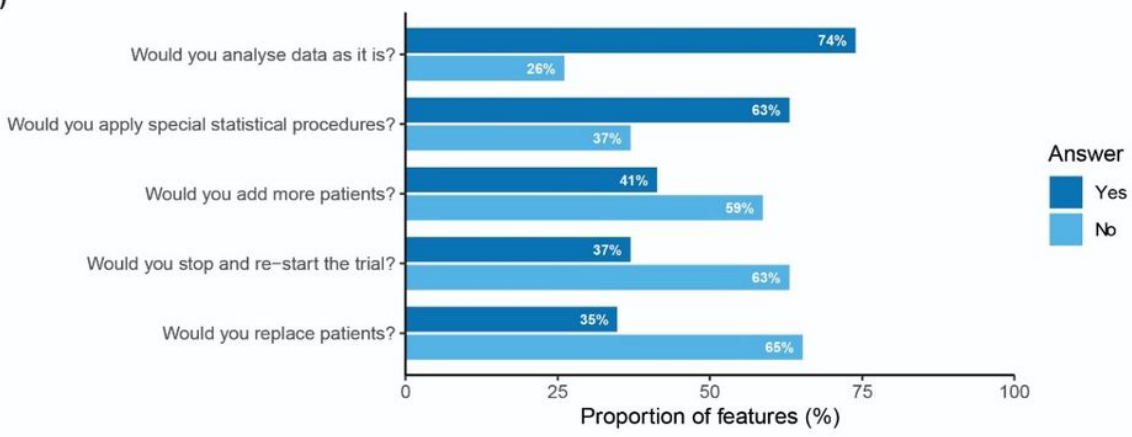

(c)

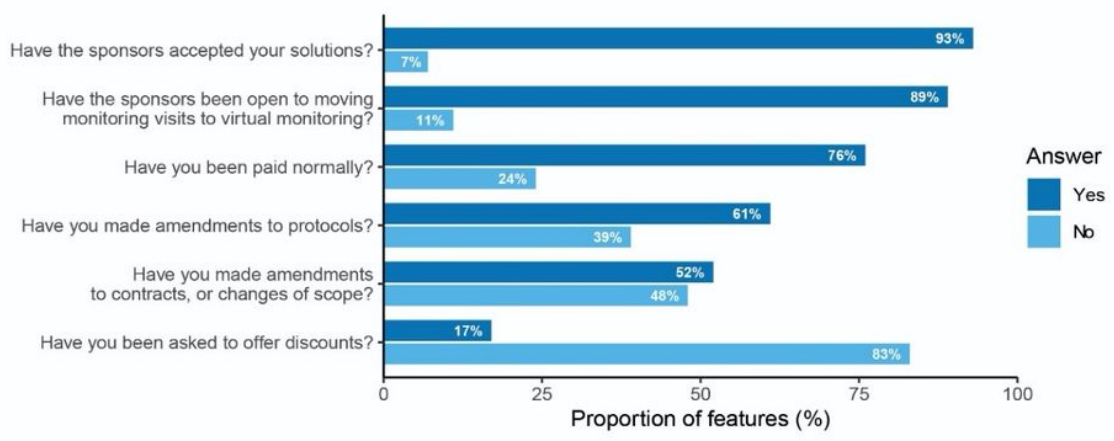

Figure 2

Estimated impact of COVID-19 on three main features in clinical trials. (a) The management of the main processes, (b) the issues related to missing data and (c) relations with the Sponsor. 

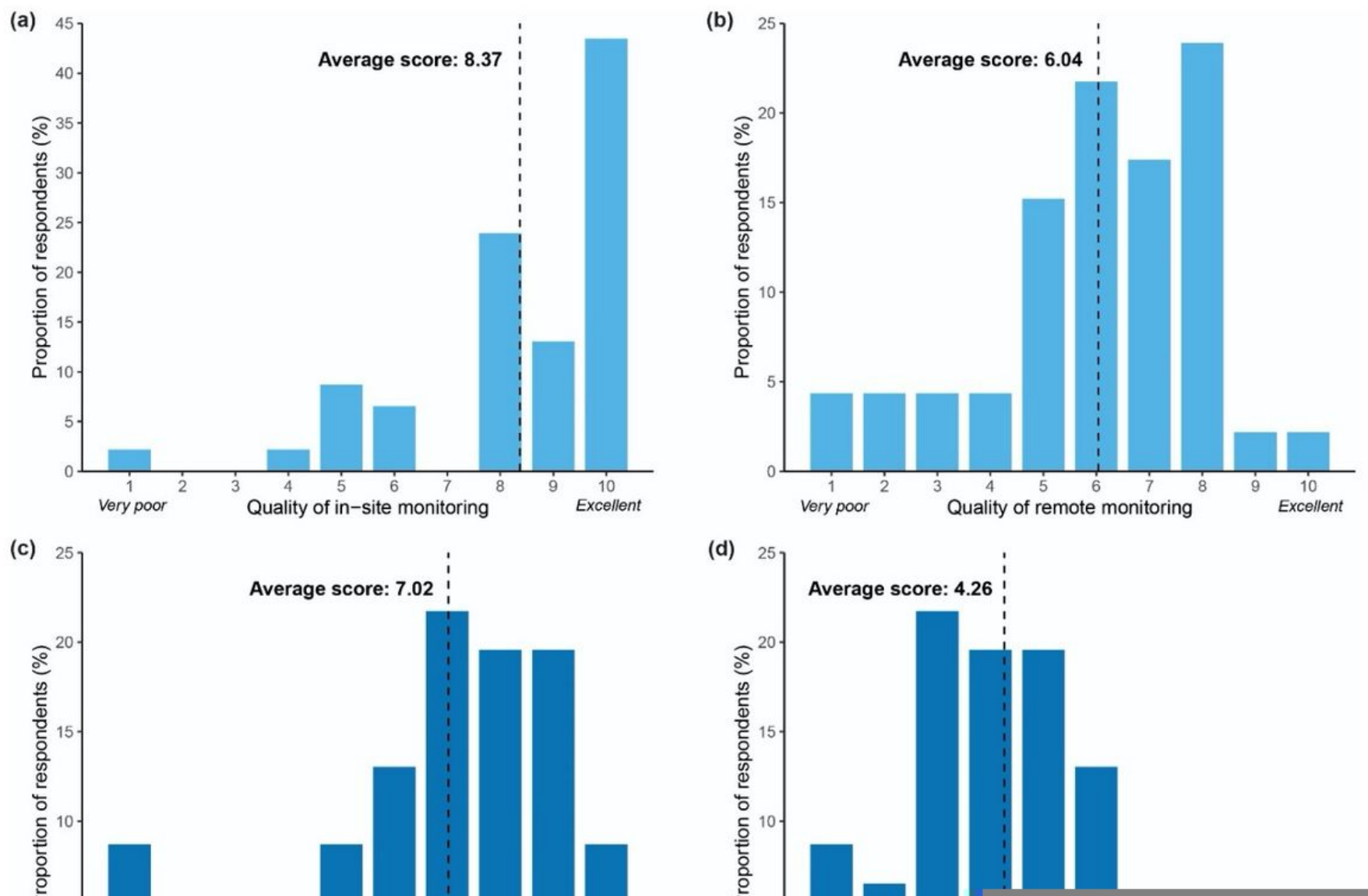

Figure 3

Estimated impact of COVID-19 on monitoring visits ranked on a 1-10 scale. Evaluation of the quality of (a) in-site monitoring and (b) remote monitoring, considering 1 as "very poor" and 10 "excellent". Evaluation of the costs of (c) in-site monitoring and (d) remote monitoring considering 1 as "very economical" and 10 "very expensive".

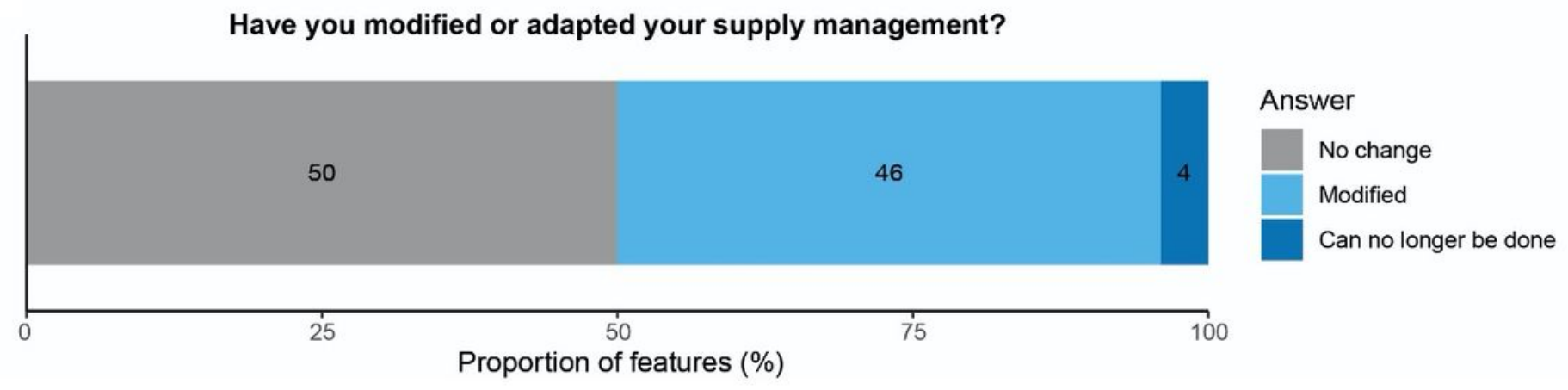

Figure 4

Estimated effect of COVID-19 on supply management from the survey. 


\section{Supplementary Files}

This is a list of supplementary files associated with this preprint. Click to download.

- CROSSChecklist.docx

- Questionnaire.docx 